\title{
IVF Outcomes with Either Highly Purified FSH vs. Recombinant FSH in Down-Regulated Normogonadotrophic Women: A Prospective Compara- tive Study in a Developing Country and Meta-Analysis
}

\author{
Mohamed K. Moustafa ${ }^{1,2}$, Ahmed R. Abdelwahed ${ }^{2}$, Ibrhium Abosekena ${ }^{2}$, Shokry Abdelazim², \\ Ahmed M. Abou-Setta ${ }^{3}$ and Hesham G. Al-Inany*,4 \\ ${ }^{I}$ Dr Erfan and Bagedo General Hospital, Jeddah, Saudi Arabia \\ ${ }^{2}$ Department of Obstetrics and Gynecology, Al-Azhar University, Cairo, Egypt \\ ${ }^{3}$ Private Clinic, Giza, Egypt \\ ${ }^{4}$ Department of Obstetrics and Gynecology, Cairo University, Cairo, Egypt
}

\begin{abstract}
Objective: In order to compare the use of highly purified (HP) follicle stimulating hormone (FSH) with recombinant FSH (recFSH) in a developing country, research should not only focus on clinical data, but also on the evidence available in the literature from previous trials.

Study Design: We performed a prospective clinical trial with 118 infertile females undergoing IVF: HP-FSH $(\mathrm{n}=59)$; recFSH $(\mathrm{n}=59)$. In addition, we performed a meta-analysis of RCTs comparing currently available HP-FSH vs. recFSH. The primary outcomes for both studies were live-birth rate and rate of ovarian hyperstimulation.

Results: In the clinical trial, the response to ovarian hyperstimulation was similar in both groups including the number of oocytes retrieved and the number of ampoules of gonadotrophins. The live birth rate per woman was $30.51 \%$ vs. $35.59 \%$ in the HP-FSH and recFSH treated groups respectively $(\mathrm{P}=0.70)$. The rate of OHSS was $5.09 \%$ in the HP-FSH compared to $6.78 \%$ in the recFSH treated groups $(\mathrm{P}=1.00)$. Regarding the meta-analysis, the live-birth $(\mathrm{O} . \mathrm{R}=1.30,95 \% \mathrm{CI}=0.92$ to 1.84$)$ and $\mathrm{OHSS}$ rates $(\mathrm{O} . \mathrm{R}=1.14,95 \% \mathrm{CI}=0.32$ to 4.04$)$ were not significantly different between the two groups. There was significantly less treatment days and total dose (IU) in the HP-FSH group compared with the rFSH group.

Conclusion: HP-FSH yields similar clinical outcome to recFSH in terms of oocytes retrieved and live-birth rate.
\end{abstract}

Keywords: Purified FSH, recombinant FSH, ICSI, pregnancy.

\section{INTRODUCTION}

Comparisons of different gonadotrophin preparations regarding efficacy and safety as well as cost effectiveness are important issues in assisted reproduction. The pregnancy rate per cycle is strongly related to the number of oocytes retrieved. On the other hand, the more oocytes stimulated the higher the risk of development of ovarian hyperstimulation syndrome.

Over the past few years, highly purified (HP) urinary products, in which the purification process allows its administration through the subcutaneous route similar to the recombinant competitors, are the latest addition to this family of infertility drugs. In clinical trials, recombinant follicle stimulating hormone (recFSH) has been proposed to be clinically more efficient than HP-FSH (Metrodin-HP ${ }^{\circledR}$ ) [1]. However, Metrodin- $\mathrm{HP}^{\circledR}$ has been withdrawn from the market and is no longer available for clinical use. It has been replaced by newer compounds with a higher concentration of glycosylated FSH $\left(\right.$ Fostimon $\left.^{\circledR}\right)$. Whether the improvements in specific activity, purity, degradation and impurities presented in

*Address correspondence to this author at the 8-Moustapha Hassanin St., Manial, Cairo, 11451, Egypt; E-mail: kaainih@yahoo.com the new generation of FSH products [2] will make them more desirable for ovarian stimulation is still unclear.

In a previous study [3] demonstrated the safety and efficacy of Fostimon ${ }^{\circledR}$ in ovulation induction comparing it to human menopausal gonadotrophin. In the present study, we wished to evaluate the efficacy of Fostimon ${ }^{\circledR}$ prospectively comparing it to recombinant FSH used in women undergoing ICSI and to perform a systematic review and meta-analysis of prospective, properly randomized trials comparing Fositmon ${ }^{\circledR}$ with recFSH.

\section{MATERIAL \& METHODS}

\section{Prospective, Controlled Clinical Trial}

The present study was designed as a prospective, controlled clinical trial to test the efficacy and safety of Fostimon ${ }^{\circledR}$ in ovulation induction for infertile pituitary suppressed females undergoing assisted reproduction.

During the period between November 2006 and June 2007, 118 infertile couples attending the IVF unit and were considered eligible were solicited for enrollment. Selection criteria for the female partner were: age between $18-39$ years old at time of recruitment, good physical health, normal menstrual cycle with a range of $24-35$ days, and no 
ovarian stimulation in the previous cycle. Exclusion criteria were: moderate to severe endometriosis, cases of endocrine abnormalities (e.g. hyperprolactinaemia), and/or failure to provide consent for enrollment. All patients had a previous complete infertility work up and allocation was performed according to clinician's choice. The hospital scientific committee approved the study and every participants signed an informed consent and the trial was performed in accordance with the Declaration of Helsinki.

Participants were down-regulated with mid-luteal gonadotrophin releasing hormone agonist (Decapeptyl, Ferring, Germany). Pituitary down regulation was confirmed by absence of follicles more than $5 \mathrm{~mm}$ and thin endometrial lining, both determined by transvaginal sonography. Ovarian stimulation was then commenced with a daily dose of HPFSH (Fostimon, IBSA, Switzerland) in the first group $(\mathrm{n}=$ 59) administered I.M. or S.C. according to patient preference. In the second group $(n=59)$ recFSH (Gonal-F, Serono, Switzerland) was administered S.C. Participants were assigned to either HP-FSH or rFSH by the first author using alternate number method. The dose for the first 5 days for both groups was 225 IU/ day. Afterwards, the dose was adjusted according to the follicular growth pattern as determined by serial transvaginal ultrasonography in correlation with the serum levels of $\mathrm{E}_{2}$. Monitoring was continued till the leading follicle reached a mean diameter of $18 \mathrm{~mm}$, at which time human chorionic gonadotrophin (10,000 IU) was administered I.M. (Choriomon, IBSA, Switzerland) to induce final oocyte maturation.

Oocyte recovery was performed via an ultrasonic guided transvaginal route 36 hours after hCG administration. The recovered oocytes were fertilized by the standard ICSI procedure and a maximum of four embryos was allowed to transfer, depending upon patient age, previous history and embryo quality. Luteal phase support was given for at least 2 weeks and included $100 \mathrm{mg}$ of progesterone daily injection I.M. Clinical pregnancy was diagnosed by the presence of an embryonic sac by US six to eight weeks post-transfer. Pregnancy termination after more than 20 weeks gestation with a living baby was considered to be a live birth.

\section{Biostatistical Analysis}

Statistical analysis was performed according to the intention to treat principle. All analyses of significance were twosided and tested at the $5 \%$ level; values of $\mathrm{P}<0.05$ were considered to indicate significant differences. Continuous variables were tested for normal distribution using the f-test. When data was found to be parametric, the results of the two groups were compared using the t-test. Qualitative variables were compared with chi-square test with Yates correction or Fisher's exact test, when necessary, and the $95 \%$ confidence intervals $(95 \% \mathrm{CI})$ using the Woolf (logit) approximation. Odds ratios (OR) and 95\% confidence intervals (95\% CI) were calculated to examine the odds of improving clinical outcomes. Clinical and demographic data are also presented as mean $( \pm \mathrm{SD})$ or as frequency distribution for simplicity. Statistical analysis was performed using the computer statistical package StatsDirect (StatsDirect Ltd, Cheshire, UK).

\section{Systematic Review and Meta-Analysis}

For the systematic review and meta-analysis, all published, unpublished and ongoing properly randomized trials reporting data that compared ovarian stimulation with Fositmon $^{\circledR}$ vs. recFSH were sought using MEDLINE (1966 to present), EMBASE (1980 - Present), the Cochrane Central Register of Controlled Trials (CENTRAL) on the Cochrane Library Issue 2, 2007, the National Research Register (NRR), and the Trial Register of Controlled Trials (www.controlled-trials.com), KoreaMed, Iranian Academic Center for Education, Culture and Research's Scientific Information Database (SID) and the Latin American \& Caribbean Health Sciences Literature database (LILACS). The following Medical Subject Headings (MeSH) and text words were used: FSH, follicle stimulating hormone, recombinant, urinary, highly purified, and randomised controlled trial(s), randomized controlled trial(s). Quasi-randomized trials were not considered to be truly randomized trials and were excluded.

The outcome measures for this systematic review were the live birth and ovarian hyperstimulation syndrome (OHSS) rates. Secondary outcomes were the clinical pregnancy, multiple pregnancy and miscarriage rates, in addition to cycle characteristics (e.g. treatment duration, number of ampoules, $E_{2}$ on day of hCG, number of oocytes retrieved).

Data management and statistical analyses were conducted using the 'Review Manager (RevMan) 4.2'. Individual outcome data were included in the analysis if they met the pre-stated criteria. Where possible, data was extracted to allow for an intention-to-treat analysis; defined as including in the denominator all randomized cycles. If data from the trial reports was insufficient or missing, the investigators of individual trials were contacted via E-mail for additional information, in order to perform analyses on an intention-totreat basis.

For the meta-analysis, the number of participants experiencing the event in each group of the trial was recorded. Meta-analysis of binary data was performed using the Mantel-Haenszel method utilizing a fixed effect model, and the odds ratio (O.R) and $95 \%$ confidence intervals $(95 \% \mathrm{CI})$, evaluated. Meta-analysis of continuous data was performed using the Weighted mean difference method utilizing a fixed effect model.

Heterogeneity by visual inspection of the outcomes tables and by using the Chi-square test $\left(\chi^{2}\right.$-test) for heterogeneity with a $10 \%$ level of statistical significance and $\mathrm{I}^{2}$ tests was utilized. The $\mathrm{I}^{2}$ test is a statistical measure used to identify and quantify heterogeneity. It describes the percentage of the variability in effect estimates that is due to heterogeneity rather than sampling error (chance) [4]. An $\mathrm{I}^{2}$ value greater than $50 \%$ may be considered to represent substantial heterogeneity. Where statistical heterogeneity was found, the reviewers looked for an explanation.

\section{RESULTS}

\section{Prospective, Controlled Clinical Trial}

A total of 118 infertile females (FSH-HP = 59 and recFSH = 59) underwent ovulation induction. All participants were Egyptians and under treatment in a developing country (Egypt). Both treatment groups were comparable in demographic and infertility characteristics (Table 1).

The response to ovarian hyperstimulation was similar in both groups. The response to ovarian hyperstimulation was 
Table 1. Characteristics of Participants in the Prospective, Clinical Trial

\begin{tabular}{|c|c|c|c|}
\hline & Fostimon & Gonal F & Significance \\
\hline Number of women & 59 & 59 & - \\
\hline Primary infertility & $67.80 \%$ & $71.19 \%$ & $\mathrm{P}=0.84$ \\
\hline Secondary infertility & $30.51 \%$ & $28.81 \%$ & $\mathrm{P}=1.00$ \\
\hline Male & $74.58 \%$ & $72.88 \%$ & $\mathrm{P}=1.00$ \\
\hline Unexplained & $16.95 \%$ & $13.56 \%$ & $\mathrm{P}=0.80$ \\
\hline Tubal & $6.78 \%$ & $13.56 \%$ & $\mathrm{P}=0.36$ \\
\hline Duration of infertility & $8.73 \pm 3.87$ & $8.95 \pm 3.52$ & $P=0.75$ \\
\hline
\end{tabular}

similar in both groups. The number of oocytes retrieved was $11.69 \pm 3.73$ following stimulation with $33.39 \pm 3.36$ ampoules of HP-FSH. The recFSH treated group required 34.10 \pm 3.82 ampoules to produce $9.25 \pm 3.42$ oocytes. The number of embryos transferred per woman $2.64 \pm 0.58$ in the Fostimon group and $2.44 \pm 0.60$ in the recFSH group. The pregnancy rate per woman was $38.98 \%$ vs. $44.07 \%$ in the Fostimon and recFSH treated groups respectively $(\mathrm{P}=0.71)$. The live-birth rate was $19 / 59$ (32.20\%) vs. $22 / 59$ (37.29\%) in the Fostimon and recFSH group groups respectively $(\mathrm{P}=$ $0.70)$. No cases of severe OHSS was encountered in either group as we routinely apply coasting for those with E2 higher than 4,000 pg/ml Table 2.

\section{Meta-Analysis of Prospective Randomized Trials}

Live-birth $(\mathrm{O} . \mathrm{R}=1.30,95 \% \mathrm{CI}=0.92$ to 1.84$)$ and OHSS rates $(\mathrm{O} . \mathrm{R}=1.14,95 \% \mathrm{CI}=0.32$ to 4.04$)$ were not significantly different between the two groups. As for the secondary outcomes, there was significantly less treatment days (WMD $=-0.27,95 \% \mathrm{CI}=-0.52$ to -0.02 ) and total dose of FSH (IU) (WMD $=-329.80,95 \% \mathrm{CI}=-483.82$ to $175.77)$ in the HP-FSH group compared with the recFSH group.

However, the number of oocytes retrieved (WMD = $0.51,95 \% \mathrm{CI}=-1.06$ to 0.04 ) was not significantly different between the two groups. In addition, there were no significant differences with regards the clinical pregnancy rate (O.R $=1.27,95 \% \mathrm{CI}=0.96$ to 1.67$)$ or the other secondary outcomes Table 3.

\section{DISCUSSION}

In contrast to developed economies, cost-effectiveness is a vital aspect of in vitro fertilization programs. In Egypt, as in many developing countries, recombinant drugs are highly

Table 2. Cycle Characteristics in the Prospective, Clinical Trial

\begin{tabular}{|c|c|c|c|}
\hline & Fostimon & Gonal F & 59 \\
\hline \hline Number of women & 59 & $34.10 \pm 3.82$ & $\mathrm{P}=0.28$ \\
\hline Number of FSH amp & $33.39 \pm 3.36$ & $21.17 \pm 1.46$ & $\mathrm{P}=0.90$ \\
\hline Duration of agonist & $21.14 \pm 1.31$ & $9.25 \pm 3.42$ & $\mathrm{P}=0.0003$ \\
\hline Number of oocytes retrieved & $11.69 \pm 3.73$ & $6.41 \pm 2.88$ & $\mathrm{P}=0.004$ \\
\hline Number of M2 & $8.17 \pm 3.52$ & $5.07 \pm 2.53$ & $\mathrm{P}=0.04$ \\
\hline Number of 2PN & $6.19 \pm 3.19$ & $2.44 \pm 0.60$ & $\mathrm{P}=0.06$ \\
\hline Number of embryos transferred & $2.64 \pm 0.58$ & $25.42 \%$ & $\mathrm{P}=0.84$ \\
\hline Difficult embryo transfer & $28.81 \%$ & $11.86 \%$ & $\mathrm{P}=1.00$ \\
\hline Blood on catheter & $10.17 \%$ & $44.07 \%$ & $\mathrm{P}=0.71$ \\
\hline Clinical pregnancy & $38.98 \%$ & $1.69 \%$ & $\mathrm{P}=1.00$ \\
\hline Miscarriage & $3.39 \%$ & & \\
\hline
\end{tabular}


Table 3. Summary Table for Meta-Analyses of HP-FSH vs. recFSH

\begin{tabular}{|c|c|c|c|c|c|c|}
\hline Comparison or outcome & $\begin{array}{c}\text { Included } \\
\text { Studies }\end{array}$ & \multicolumn{2}{c|}{$\begin{array}{c}\text { Number of } \\
\text { Participants }\end{array}$} & Statistical method & Effect size \\
\hline \hline \multicolumn{7}{|c|}{ Pregnancy Outcomes (HP-FSH versus recFSH) } \\
\hline 01 Number of embryos obtained & 1 & 151 & WMD (fixed), 95\% CI & $0.10[-1.13,1.33]$ & - \\
\hline 02 Number of embryos transferred & 2 & 408 & WMD (fixed), 95\% CI & $-0.19[-0.39,0.01]$ & $0.00 \%$ \\
\hline 03 Clinical Pregnancy Rate & 5 & 1004 & OR (fixed), 95\% CI & $1.27[0.96,1.67]$ & $0.00 \%$ \\
\hline 04 Multiple Pregnancy Rate & 1 & 267 & OR (fixed), 95\% CI & $1.75[0.79,3.86]$ & - \\
\hline 05 Miscarriage rate & 3 & 701 & OR (fixed), 95\% CI & $1.13[0.57,2.25]$ & $0.00 \%$ \\
\hline 06 Ongoing pregnancy rate & 2 & 434 & OR (fixed), 95\% CI & $1.24[0.76,2.01]$ & $0.00 \%$ \\
\hline 07 Ongoing pregnancy/ live-birth rate & 4 & 853 & OR (fixed), 95\% CI & $1.25[0.92,1.71]$ & $0.00 \%$ \\
\hline 08 Live-birth rate & 3 & 596 & OR (fixed), 95\% CI & $1.30[0.92,1.84]$ & $0.00 \%$ \\
\hline
\end{tabular}

expensive; hence commercially available gonadotrophins are predominantly urinary derived. Therefore for a change in prescription policies to occur, more effective and efficient treatment options need to be developed as compared to the currently used medications.

In the present clinical trial, there was no statistically significant difference between both groups regarding the numbers of retrieved oocytes at time of ovum pickup although the Fostimon-treated group was slightly higher. The same observation was found in the number of ampoules, but the number was slightly lower in the FSH-HP treated group.

Pregnancy rate was considered as a secondary endpoint as they rather depend on the number of embryos replaced. There was no significant difference in the number of embryos replaced indicating the adherence to the protocol of ovulation induction was similar in both groups. Clinical pregnancy rate was not statistically different between both groups. The non-statistically significant difference in clinical pregnancy rate between the two gonadotrophin preparations was matching with other reported trials in the medical literature $[5,6]$.

Miscarriage rate was also considered as a secondary endpoint and although there was no statistically significant difference between both groups but there it was lower in the recFSH treated group.

The most important side effect of gonadotrophin treatment in ovarian stimulation is the occurrence of OHSS. Thus, safety endpoint of the present trial included the incidence of ovarian hyperstimulation syndrome and multiple pregnancies. There was no statistically significant difference in the incidence of OHSS in both groups as no cases developed severe OHSS, however, the incidence of severe hyperstimulation requiring hospitalization is so low that the power of the study would be insufficient to detect a significant difference between the two groups. There was no recorded seri- ous local reaction from either FSH-HP or recFSH treated groups.

This study can be criticized of being non-randomized and of its limited sample size. Based on our results from the clinical trial, for a study power of $80 \%$, it would require the enrolment of 960 subjects, a task practically impossible for a single IVF unit to complete in a reasonable period of time. It is clear that our results require further validation through a multi-center trial or by means of a meta-analysis of studies similar to ours. Even so, overall it can be concluded that the clinical outcome was not different between the purified and recombinant FSH treated groups.

The clinical outcomes of the clinical trial are also in line with the results of the meta-analysis of prospective, randomized trials. This meticulous systematic review demonstrated that the two currently available preparations are not significantly different with regards the probability of a live-birth or the development of OHSS.

In previous systematic reviews comparing recombinant FSH and urinary FSH, there was no apparent trend in outcomes with one review team claiming superiority of recombinant FSH [1, 7] while the other concluding that there is insufficient evidence to determine if either is more favorable [8]. This is the result of several differences in the preparations that were available then, compared with today's available products. That is why we decided to concentrate on only commercially available products to determine the best available source today.

The technological developments of gonadotrophins over the last ten years have shown improvements in specific activity, purity, degradation and impurities. This has been reflected with the production of highly purified FSH preparations. In addition, a clinically oriented value of FSH isoforms is of importance for clinical efficacy as it may affect the developmental competence of oocytes [9]. Moreover, the FSH isoforms in Metrodin-HP ${ }^{\circledR}$ has been documented to be 
of lower activity than expected [10], and subsequently this product has been removed from the market.

Lastly, since there are differences in the costs and efficiencies associated with each stimulation therapy, a costeffective analysis should be performed according to regional prices in order to ascertain if a change in policy should be enforced.

\section{CONDENSATION}

In a clinical trial and meta-analysis of RCTs, HP-FSH yields similar to recFSH in terms of oocytes retrieved and live-birth rate.

\section{REFERENCES}

[1] Nayudu PL, Vitt UA, Barrios De Tomasi J, Pancharatna K, UlloaAguirre A. Intact follicle culture: what it can tell us about the roles of FSH glycoforms during follicle development. Reprod Biomed Online 2002; 5(3): 240-53.

[2] Daya S, Gunby J. Recombinant versus urinary follicle stimulating hormone for ovarian stimulation in assisted reproduction. Hum Reprod 1999; 14(9): 2207-15.

[3] Baker VL, Fujimoto VY, Kettel LM, et al. Clinical efficacy of highly purified urinary FSH versus recombinant FSH in volunteers undergoing controlled ovarian stimulation for in vitro fertilization: a randomized, multicenter, investigator-blind trial. Fertil Steril $2008 ; 24$.

[4] Grudzinskas G. Recent clinical evidence for selecting u-FSH or rFSH in ART. Fertil Steril 2006; 86(3): S427-S28.

[5] Andersen CY, Westergaard LG, van Wely M. FSH isoform composition of commercial gonadotrophin preparations: a neglected aspect? Reprod Biomed Online 2004; 9(2): 231-6.

[6] Foutouh IA, Khattab S, Mohesn IA, Moaz M, Al-Inany H. Clinical outcome following stimulation with HMG versus highly purified HMG in patients undergoing ICSI. Reprod Biomed Online 2007; 14(2): 145-7.

[7] Bassett RM, Driebergen R. Continued improvements in the quality and consistency of follitropin alfa, recombinant human FSH. Reprod Biomed Online 2005; 10(2): 169-77.

[8] Daya S. Updated meta-analysis of recombinant follicle-stimulating hormone (FSH) versus urinary FSH for ovarian stimulation in assisted reproduction. Fertil Steril 2002; 77(4): 711-4.

[9] Bayram N, van Wely M, van der Veen F. Recombinant FSH versus urinary gonadotrophins or recombinant FSH for ovulation induction in subfertility associated with polycystic ovary syndrome. Cochrane Database Syst Rev 2001; 2: CD002121.

[10] Higgins JP, Thompson SG, Deeks JJ, Altman DG. Measuring inconsistency in meta-analyses. BMJ 2003; 327(7414): 557-60.

(c) Moustafa et al.; Licensee Bentham Open.

This is an open access article licensed under the terms of the Creative Commons Attribution Non-Commercial License (http://creativecommons.org/licenses/by-nc/3.0/) which permits unrestricted, non-commercial use, distribution and reproduction in any medium, provided the work is properly cited. 